\title{
Are sexually transmitted infections increasing in adolescents?
}

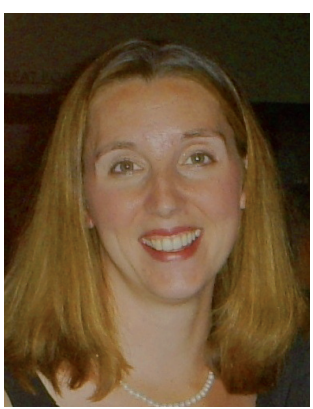

Jessica M Sales Emory University, Rollins School of Public Health, 1520 Clifton Road NE, Room 266, Atlanta, GA 30322, USA Tel.: +1 4047276598 ; Fax: + 1404727 1369; E-mail:jmcderm@emory.edu

\author{
'In 2008 ... one in four adolescent \\ girls (aged between 14 and \\ 19 years) in the USA are infected \\ with one of four common sexually \\ transmitted infections.'
}

In 2008, to most, it is not shocking news to hear that adolescents are having sex. However, in recent months, it may have shocked many to hear findings from a highly publicized national study stating that one in four adolescent girls (aged between 14 and 19 years) in the USA are infected with one of four common sexually transmitted infections (STIs) [1]. For those working in areas related to adolescent sexual health, these statistics were not likely surprising. For over a decade, compared to older adults, adolescents (15- to 19-years old) and young adults (20- to 24-years old) have had disproportionately higher rates of STIs [2]. The striking rate of STIs in the adolescent population often begets two questions: are STIs increasing in adolescents and why are STI rates so high among this population? Here, I provide a brief overview of adolescent sexual behaviors, the current status of STI and HIV rates in adolescents, and a summary of adolescent STI surveillance data compiled over the past 20 years, to assess whether STIs are increasing in adolescents, as well as providing some potential explanations for why STI rates are so high in the adolescent population.

\section{Sexual behavior during adolescence}

Many adolescents and young adults engage in sexual intercourse and, often at times, with multiple partners and without using condoms. In $2001,45.6 \%$ of high school students in the USA [3], and $79.5 \%$ of college students (aged 18-24 years) [4], have had sexual intercourse. Although most adolescents do not have simultaneous sexual partners at any given point in time, the number of sexual partners adds up over time. Specifically, among high school seniors in 2001, approximately $22 \%$ reported having sex with at least four different partners [3]. Moreover, among sexually active adolescents, only 58\% reported using a condom the last time they had intercourse [3]. In spite of the fact that many adolescents have used condoms during some episodes of sexual intercourse, comparatively few report using them every time they have sex [5]. Thus, adolescents engage in sexual behaviors that place them at risk for acquiring STIs, including HIV.

\section{STIs/HIVIAIDS \& adolescents:} the extent of the problem

Globally, over 100 million STIs occur each year in people under 25 years of age [6], and an estimated 11.8 million people aged between 15 and 24 years were living with HIV by mid-2002. Furthermore, approximately half of all new HIV infections worldwide, or nearly 6000 cases per day, occur in young people [7].

\section{....over 100 million STIs occur each year in people under 25 years of age, and an estimated 11.8 million people aged between 15 and 24 years were living with HIV by mid-2002.'}

In the USA alone, approximately one quarter of new STI infections, almost 4 million, are diagnosed in teenagers $[8,9]$. Moreover, approximately 1700 newly diagnosed cases of AIDS were reported in people between the ages of 13 and 24 years in 2003 [10]. However, the risk of STI/HIV infection is not uniform among adolescents. Females, men who have sex with men, injecting drug users, people who have exchange sex (i.e., sex for money or goods), and racial minorities have markedly higher rates of STI/HIV during adolescence [11].

\section{Are STIs increasing in adolescents?}

Over the past 20 years, the CDC have compiled adolescent STI surveillance data on three nationally notifiable STIs (chlamydia, gonorrhea and syphilis). Reports have been published of these data statistics and trends for chlamydia, gonorrhea and syphilis in the adolescent population in the USA from the 1980 s to 2006 [2,11]. The surveillance information in these reports are based on the following sources of data: 
- Case reports from state and local STI programs;

- Regional Infertility Prevention Projects;

- The National Job Training Program;

- The Corrections STD Prevalence Monitoring Project;

- The Men Who Have Sex With Men (MSM) Prevalence Monitoring Project;

- The Gonococcal Isolate Surveillance Project;

- National surveys implemented by federal and private organizations.

Case report data for other STIs are not presented because they are not nationally notifiable diseases. However, the CDC STI surveillance reports provide the most comprehensive data on STI rates in adolescents available and also allows for the examination of trends in STI rates (i.e., increases or decreases in chlamydia, gonorrhea and syphilis) in this population over the past two decades. Each infection will now be examined in turn.

'From 2005-2006, adolescents and young adults had the highest rates of gonorrhea compared with any other age groups.'

\section{Chlamydia rates}

For all age groups in 2006, 1,030,911 chlamydial infections were reported to the CDC from all 50 states and the District of Columbia, USA. Rates of nationally reported chlamydia infections among women have been increasing annually since the early 1990s when public programs for screening and treatment were established. From 1987-2006, rates of chlamydial infection in women (all ages) increased from 78.5 cases to 515.8 cases per 100,000 population. These increases are believed to be due largely to increased chlamydia screening, the implementation of more sensitive tests to detect infection (i.e., nucleic acid amplification tests), and improved reporting, as well as the continuing high prevalence of disease [2].

Specific to the female adolescent/young adult population (15-24 years of age), in 2006, the median state-specific chlamydia test positivity rate was $6.7 \%$ (range: 2.8 to $16.9 \%$ ) in all 50 states, the District of Columbia, Puerto Rico and the Virgin Islands [12]. Examination of female adolescent/young adult chlamydia infection rates over time indicate that infection rates have remained fairly stable, with only small fluctuations from year-to-year in the rate of disease. No comparable data exist for adolescent males, but due to the increased availability of urine testing, more males are now tested for chlamydia [101]. Thus, from 2002-2006, the chlamydia rate in men increased by $36 \%$ in comparison with a $16 \%$ increase in women over the same timeframe [2].

\section{Gonorrhea rates}

Following a nearly $75 \%$ decrease in the rate of reported gonorrhea from 1975 to 1997 , for two consecutive years (2005-2006) gonorrhea rates for adolescents (15- to 19-years) and young adults (20- to 24-years) increased by 6.3 and $4.4 \%$, respectively. In the period of 2005 to 2006, adolescents and young adults had the highest rates of gonorrhea compared with any other age groups [2].

\section{Syphilis rates}

In the overall population, the rate of primary and secondary syphilis declined by nearly $90 \%$ from 1990 to 2000 to their lowest rates since national syphilis reporting began in 1941 . However, since 2001, rates of syphilis have increased each year, mostly in males, but also in females too. For adolescent females (15-19 years of age), rates have increased since 2004 through the last published reporting year of 2006. Moreover, young adult women (20-24 years of age), have had the highest rates of syphilis for all age groups since 2002 . Specific to adolescent males, syphilis infections have also increased since 2002, but rates are still highest amongst males aged 35 to 39 years [2].

\section{Interpreting the observed increases}

From the CDC surveillance data, it appears that STI rates, at least for chlamydia, gonorrhea and syphilis, are indeed increasing in the adolescent population in the USA.

\section{....adolescents may be ill informed about STIs, their symptoms, the need for treatment, and where and how they can obtain treatment.'}

However, the extent to which these observed increases are a true reflection of increased disease burden in adolescents or the byproduct of increased testing and diagnosis among adolescents is yet to be determined. Likely, it is a combination of both. 


\section{Explanations for high STI rates in adolescents}

Adolescents are at especially high risk of contracting STIs, including HIV, for several reasons.

\section{Immature biology}

Biologically, adolescents' immature reproductive and immune systems make them more vulnerable to infection by various STI agents [13]. For example, adolescent women may have a physiologically increased susceptibility to infection due to increased cervical ectopy [11].

\section{Earlier sexual debut \& later onset of marriage}

Over the past few decades, both the onset of puberty and the initiation of sexual intercourse have occurred at decreasing ages [14], yet the age at first marriage has increased, with the median age of first marriage at 28.6 years for men and 26.6 years for women [15].

\section{'...adolescent women may have a physiologically increased susceptibility to infection due to increased cervical ectopy.'}

Thus, adolescents usually have had sexual intercourse with multiple partners prior to marriage, thereby increasing their chances of acquiring an STI or HIV.

\section{Contextual conditions}

Conditions such as poverty, homelessness, political strife and dislocation, all problems that are increasingly common among adolescents in developing countries, are associated with sexual abuse or sexual intercourse in exchange for money or support for basic needs [16]. In addition, for social and cultural reasons, adolescents, especially young girls, may be less able to refuse sex and/or less able to insist on adequate protection, thereby increasing their risk for STI/HIV infection [17].

\section{Barriers to quality STI-prevention services}

Often, adolescents are confronted with multiple barriers to seeking and receiving STI testing and treatment, such as lack of insurance, lack of money to pay, lack of transportation, discomfort with facilities and services designed for adults, and concerns about confidentiality [11]. Moreover, adolescents may be ill informed about STIs, their symptoms, the need for treatment, and where and how they can obtain treatment.

\section{Conclusion}

Sexually transmitted infections and HIV infections in adolescents are at epidemic levels worldwide. As long as adolescents continue to engage in sexual behaviors that place them at risk for STI/HIV (e.g., sex without a condom and with multiple partners) they will be susceptible to such infections. For reasons outlined above, a few of which are amenable to change, adolescents may be especially vulnerable to STIs. It is a public health imperative that we continue to search for ways to protect our youth, as well as teach them to protect themselves, from STI/HIV infections.

\section{Financial \& competing interests disclosure}

The author has no relevant affiliations or financial involvement with any organization or entity with a financial interest in or financial conflict with the subject matter or materials discussed in the manuscript. This includes employment, consultancies, honoraria, stock ownership or options, expert testimony, grants or patents received or pending, or royalties.

No writing assistance was utilized in the production of this manuscript.

\section{Bibliography}

1. Forhan SE, Gottlieb SL, Sternberg MR et al: Prevalence of sexually transmitted infections and bacterial vaginosis among female adolescents in the United States: data from the National Health and Nutrition Examination Survey (NHANES) 2003-2004. Paper presented at: The National STD Prevention Conference, Chicago, IL, USA, March (2008).

2. Centers for Disease Control and Prevention: Sexually transmitted disease surveillance, 2006. US Department of Health and Human Services, Atlanta, GA, USA (2007).
3. Grunmaum JA, Kann L, Kinchen SA, Williams B, Ross JG, Lowry R:

Youth risk behavior surveillance, United States, 2001. MMWR 51(SS-4), 1-64 (2002).

4. Centers for Disease Control and Prevention: Youth risk behavior surveillance, United States, 1995. MMWR 46(SS-6), 1-56 (1997).

5. Moore KA, Driscoll AK, Lindberg LD: A statistical portrait of adolescent sex, contraception, and childbearing. National Campaign to Prevent Teen Pregnancy, Washington, DC, USA (1998).
6. World Health Organization: Fact Sheet No. 186. Young people and sexually transmitted diseases (1997).

7. Joint United Nations Programme on HIV/AIDS (UNAIDS): Report on the global HIV/AIDS epidemic. Geneva, Switzerland (UNAIDS publication 02.26E) (2002).

8. Centers for Disease Control and Prevention: Tracking the hidden epidemics: trends in STIs in the United States. US Department of Health and Human Services, Atlanta, GA, USA (2000). 
9. NIAID: Sexually transmitted diseases statistics. National Institute of Allergy and Infectious Diseases, US Department of Health and Human Services, Washington DC, USA (1997).

10. Centers for Disease Control and Prevention: Young people at risk: HIV/AIDS among America's youth. US Department of Health and Human Services, Atlanta, GA, USA (2003).

11. Centers for Disease Control and Prevention: Sexually transmitted disease surveillance, 2003. US Department of Health and Human Services, Atlanta, GA, USA (2004).

12. Centers for Disease Control and Prevention: Sexually transmitted disease surveillance 2006 Supplement, Chlamydia Prevalence Monitoring Project Annual Report 2006. US Department of Health and Human Services, Atlanta, GA, USA (2008).
13. Cates W, McPheeters M: Adolescents and sexually transmitted diseases: current risks and future consequences. Prepared for: the Workshop on Adolescent Sexuality and Reproductive Health in Developing Countries: Trends and Interventions. National Research Council, Washington, DC, USA (1997).

14. Sales JM, Irwin Jr CE: Theories of adolescent risk-taking: a biopsychosocial model. In: Adolescent Health: Understanding and Preventing Risk Behaviors and Adverse Health Outcomes. DiClemente R, Crosby R (Eds). (2008) (In Press).

15. Schoen R, Standish N: The retrenchment of marriage: results from marital status life tables for the United States, 1995. Popul. Develop. Rev. 27, 553-563 (2001).
16. Barker G, Fontes M: Review and analysis of international experience with programs targeted on at-risk youth. LASHC Paper Series No. 5. World Bank, Washington, DC, USA (1996).

17. Wingood GM, DiClemente RJ: Application of the theory of gender and power to examine HIV related exposures, risk factors and effective interventions for women. Health Education \& Behavior 27, 539-565 (2000).

\section{Website}

101. Centers for Disease Control and Prevention: Male chlamydia screening consultation, Atlanta, GA, USA 28-29 May, 2006. Meeting Report, 22 May, 2007. www.cdc.gov/std/chlamydia/ChlamydiaScre ening-males.pdf

(Accessed 11 July, 2008) 